\title{
NIF-0096141-OA Prop Simulations of NEL PBRS Measurements
}

C. C. Widmayer, K. R. Manes

February 21, 2003

U.S. Department of Energy

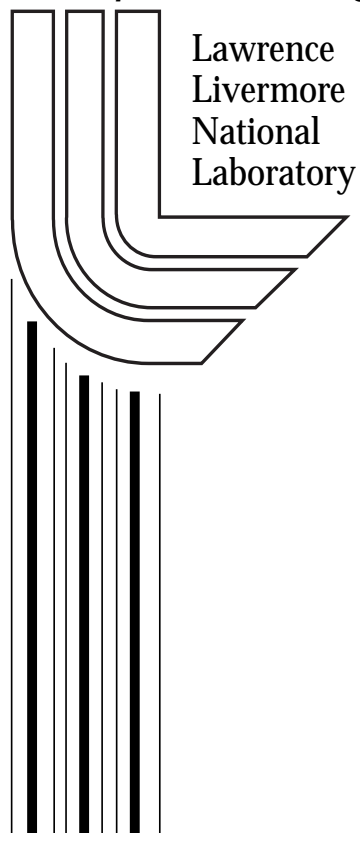




\section{DISCLAIMER}

This document was prepared as an account of work sponsored by an agency of the United States Government. Neither the United States Government nor the University of California nor any of their employees, makes any warranty, express or implied, or assumes any legal liability or responsibility for the accuracy, completeness, or usefulness of any information, apparatus, product, or process disclosed, or represents that its use would not infringe privately owned rights. Reference herein to any specific commercial product, process, or service by trade name, trademark, manufacturer, or otherwise, does not necessarily constitute or imply its endorsement, recommendation, or favoring by the United States Government or the University of California. The views and opinions of authors expressed herein do not necessarily state or reflect those of the United States Government or the University of California, and shall not be used for advertising or product endorsement purposes.

This work was performed under the auspices of the U. S. Department of Energy by the University of California, Lawrence Livermore National Laboratory under Contract No. W-7405-Eng-48.

This report has been reproduced directly from the best available copy.

Available electronically at http://www.doc.gov/bridge

Available for a processing fee to U.S. Department of Energy

And its contractors in paper from

U.S. Department of Energy

Office of Scientific and Technical Information

P.O. Box 62

Oak Ridge, TN 37831-0062

Telephone: (865) 576-8401

Facsimile: (865) 576-5728

E-mail: reports@adonis.osti.gov

Available for the sale to the public from

U.S. Department of Commerce

National Technical Information Service

5285 Port Royal Road

Springfield, VA 22161

Telephone: (800) 553-6847

Facsimile: (703) 605-6900

E-mail: orders@ntis.fedworld.gov

Online ordering: http://www.ntis.gov/ordering.htm

OR

Lawrence Livermore National Laboratory

Technical Information Department's Digital Library

http://www.llnl.gov/tid/Library.html 
To: $\quad$ Com/Ops, BLIP, ICCS, Laser diagnostics

From: $\quad$ Clay Widmayer and Ken Manes

Subject: Prop Simulations of NEL PBRS measurements.

Portable Back Reflection Sensor, PBRS, (NEL only) and Quad Back Reflection Sensor, QBRS, time delay reflectometer traces are among the most useful diagnostics of NIF laser status available. NEL PBRS measurements show several signals reaching the detector for each shot. The time delay between signals suggests that the largest of these is due to energy at the spatial filter pinhole planes leaking into adjacent pinholes and traveling back upstream to the PBRS. Prop simulations agree with current PBRS measurements to within 50\%. This suggests that pinhole leakage is the dominant source of energy at the PBRS. However, the simulations predict that the energy leakage is proportional to beam output energy, while the PBRS measurements increase more slowly ("saturate"). Further refinement of the model or the measurement may be necessary to resolve this discrepancy.

\section{PBRS data}

PBRS measurements for four NEL shots are shown in figure 1. The four shots occurred during the December shot campaign and ranged in output energy between about $1 \mathrm{~kJ}$ and $11 \mathrm{~kJ}$. (The fifth curve, in blue, is just the $5 \mathrm{~kJ}$ data scaled). Each trace shows four signals, arriving at different times, with the largest signal occurring earliest at about $1100 \mathrm{~ns}$.

Beam 315 PBRS Data for 0.8, 3.3,and $4.8 \mathrm{~kJ}$, and 10.8kJ shots

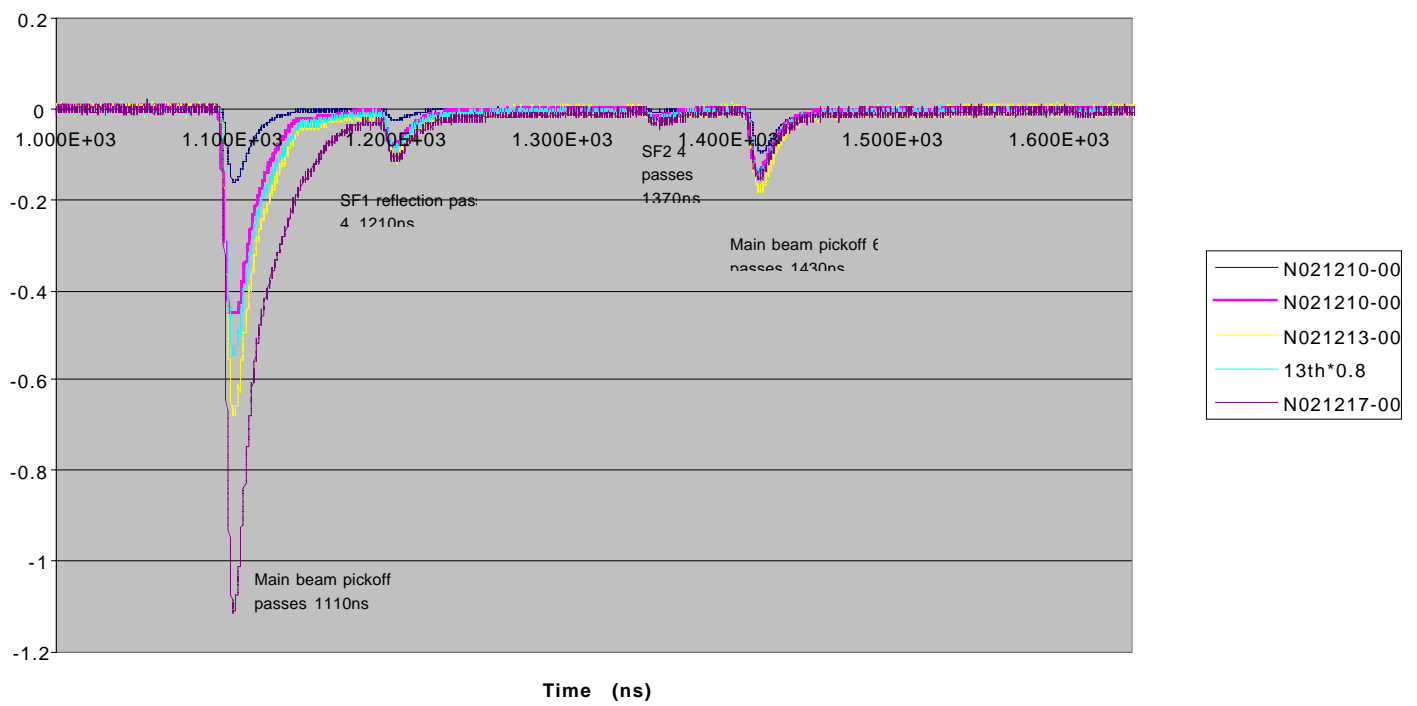

Figure 1. PBRS measurements for four NEL system shots showing a large signal occurring $1100 \mathrm{~ns}$ into each trace. 
By analyzing the propagation time through the NIF chain and the time delays between signals, Stacie Hvisc was able to identify a possible mechanism for each blip in the trace. In this way, the signal of interest here, the large one, has been attributed to pinhole leakage at the spatial filter pinhole planes. The other signals are thought to be due to 'pencil beam' reflections off of SF1 and SF2 spatial filter lenses, and leakage through the PEPC/Polarizer after switch-out. A diagram showing each of the possible back reflection mechanisms is shown in figure 2 . The propagation times for each mechanism agree quite well with the spacing of the blips in figure 1 .

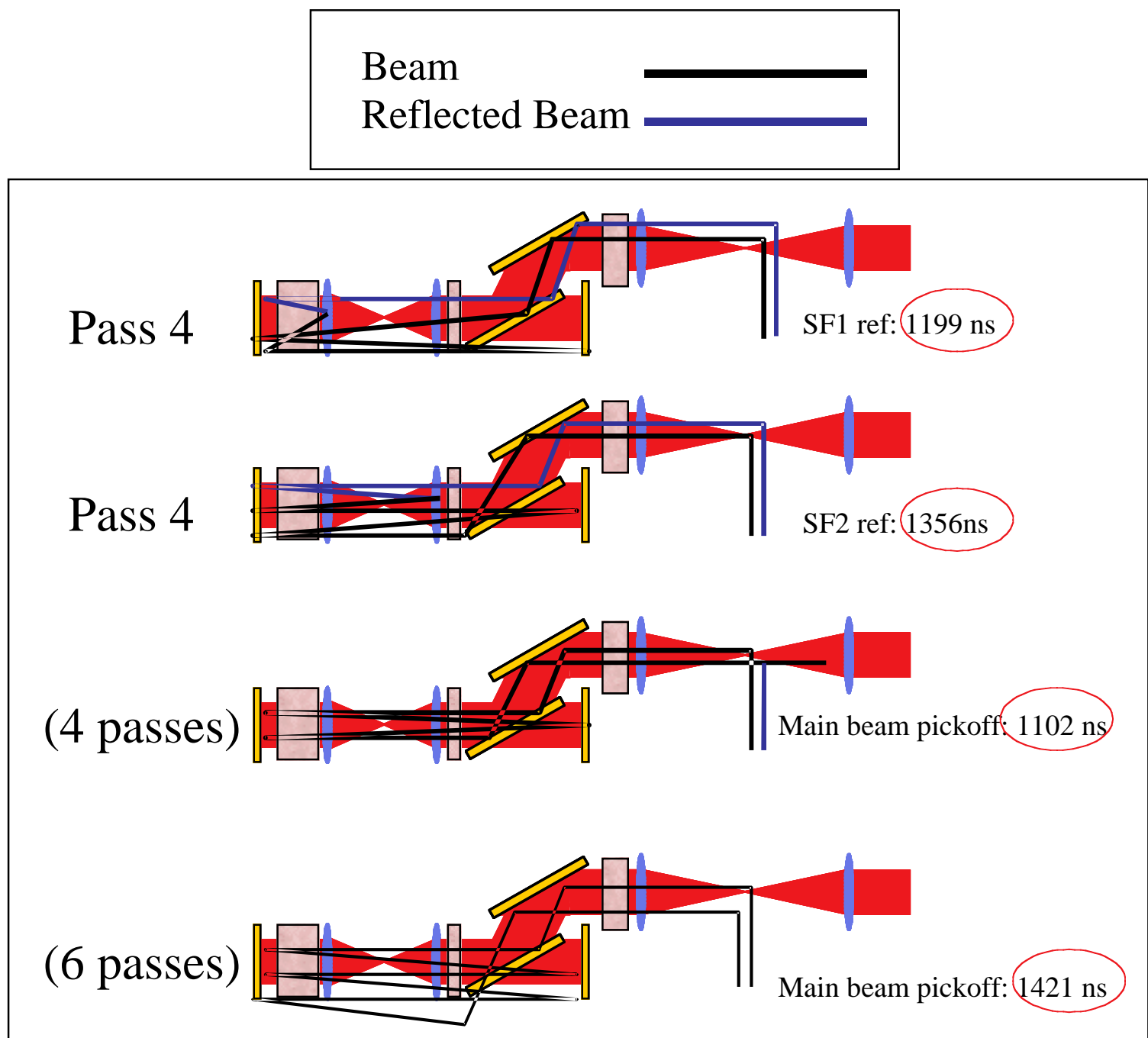

Figure 2. Possible mechanisms for NEL PBRS signals: 1) Back reflection off of SF1. 2) Back reflection off of SF2.3) Energy leaks through wrong spatial filter pinhole and goes upstream. 4) Beam leaks through Polarizer on switch-out and goes upstream.

\section{Prop92 Modeling of Pinhole Leakage}

The pinhole leakage mechanism is shown in more detail in Figure 3. The figure shows that at each spatial filter pinhole, light can leak through an adjacent pinhole, propagate backwards through the system, accumulate gain through the amplifiers, and arrive simultaneously with the other leakage signals at the PBRS. 


\section{"Cross-talk" scattering accumulates down a NIF beamline}

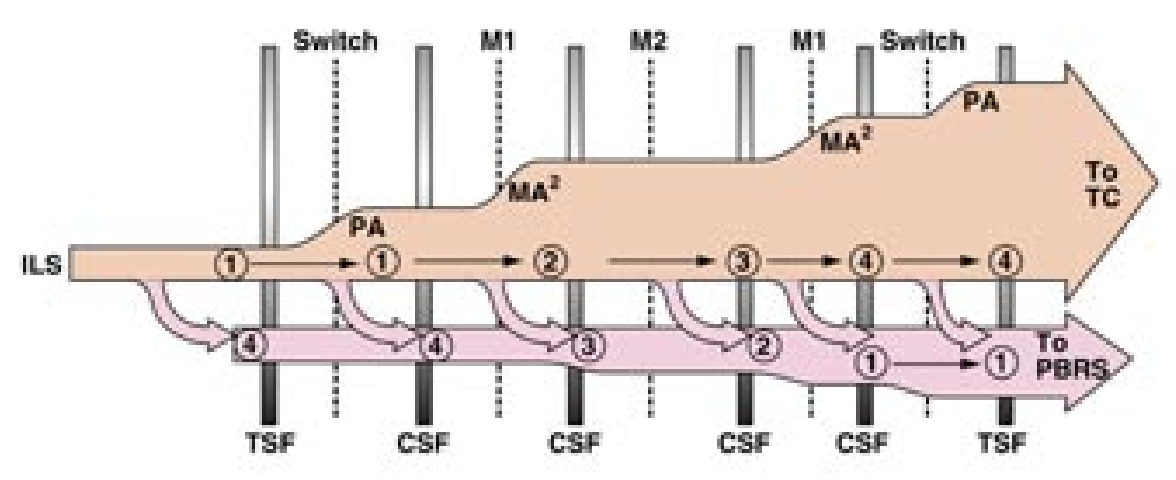

- Signal to TC is

$$
E_{1 L S} \cdot G_{P A} \cdot G_{M A}^{4} \cdot G_{P A}=E_{\text {OUT }}
$$

- Signal to PBRS is

$$
E_{\text {PBAS }}=E_{\text {OUT }}\left(F_{1-4}^{\text {ILS }}+2 F_{1-4}^{\text {PA }}+2 F_{1-4}^{M A}+2 F_{2-3}^{M A}\right)
$$

ontras

Figure 3. Diagram of pinhole leakage mechanism. The first blip on the PBRS trace is believed to be due to the sum of energy leaking to adjacent pinhole at each spatial filter pass in the system.

The NIF project was alerted to watch for cross-talk by J. P. Leidinger of CEA's LIL project. LIL back-reflection traces have been dominated by a strong cross-talk signal whose high amplitude still puzzles us. LIL has been forced to its reduce input aperture half angle from 150 microradians to 60 microradians with a significant loss of beam area. To simulate the phenomenon with Prop92, we used the NEL Living Model at increased resolution. Typically, the Living Model uses a $50 \mathrm{~cm}$ square grid with 512 points. This allows it to simulate spatial frequencies up to about $0.5 \mathrm{~mm}^{-1}$, or scattered ray angles up to 500 microrad. Since NIF pinholes are located $1 \mathrm{mrad}$ apart, we had to increase the resolution of the calculation $4 \mathrm{X}$ to $2048 \times 2048$ to model the scattered light that leaks through the neighboring pinholes. Because of the increased spatial resolution required, it was necessary to sacrifice resolution in other parts of the calculation. Consequently, these simulations were done with a single time slice and spatially flat small-signal gain profiles.

The resolution of the measured phase aberrations used in the Living Model only goes up to spatial frequencies of $0.5 \mathrm{~mm}^{-1}$ as mentioned above. Unfortunately, we do not have a complete set of measured PSD2 phase aberrations for spatial frequencies above this point. So, to simulate the higher frequency scattered light leaking through the pinhole, we used the approach employed in sub-aperture patch modeling, which is to use the mathematically synthesized "reference" aberrations developed by Mark Henesian. The reference aberrations assume that the scattered power spectrum obeys $v^{-2.55}$ power law in PSD space, but the Fourier components are randomized to produce a unique phase map 
on each optical surface. The reference aberrations were only used in the portion of the spectrum corresponding to the increased resolution: $0.5-2 \mathrm{~mm}^{-1}$. All the standard NEL Living Model phase aberrations were retained for spatial frequencies below $0.5 \mathrm{~mm}^{-1}$.

The reference aberrations used here was based on the $\langle\mathbf{x}\rangle$ model, where the amplitude of the aberrations is scaled to match the measured rms wavefront error of NIF optics in the PSD2 band between 0.4 and $8 \mathrm{~mm}^{-1}$. Because the PSD2 metrology is taken over small patches on each optical surface, the aberrations are scaled to the average of many measurements. The original data for the $\langle\mathbf{x}\rangle$ model was supplied by Chris Stolz and Tom Parham. For this study, the rms value used for amplifier slabs was increased from 0.25 $\mathrm{nm}$ rms to $0.35 \mathrm{~nm}$, based on new data from Tayyab Suratwala. Other values, such as $0.73 \mathrm{~nm}$ for spatial filter lenses, $0.5 \mathrm{~nm}$ for windows, were unchanged. For comparison, the NIF spec for the PSD2 band is $1.6 \mathrm{~nm} \mathrm{rms}$.

\section{Results}

Using this model, the far-field distribution was calculated at each pinhole plane for $1 \omega$ energies between 1 and $11 \mathrm{~kJ}$. The portion of energy striking the 200 microrad half-angle pinhole located $1 \mathrm{mrad}$ from the focal spot was calculated and multiplied by the gain of the rest of the system to simulate propagation to the PBRS. The total leakage, summed over all pinhole passes is interpreted as the energy reaching the PBRS. The results are shown in figure 4.

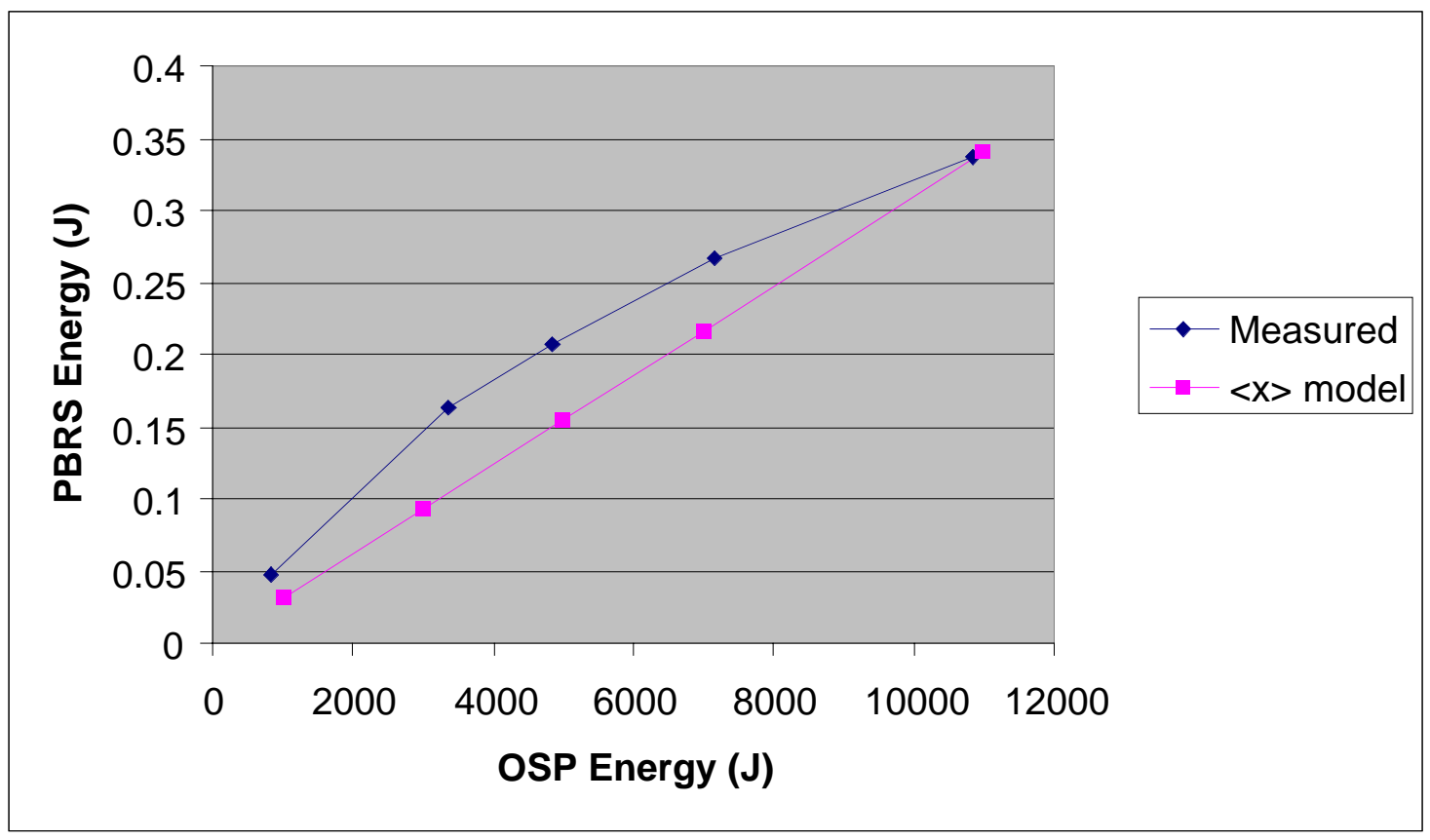

Figure 4. Measured PBRS energy (blue) and Prop calculation (red).

From the figure, the Prop92 calculation agrees to within 50\% with the PBRS measurement over the range of output energies studied. We contend that this agreement is not bad considering how sensitive the back reflection estimate is on PSD2 rms, and that PSD2 is probably the most incompletely characterized portion of the entire NIF wavefront. For example, a $50 \%$ disagreement in scattered power implies a $22 \%$ 
disagreement in rms wavefront error ( $\operatorname{sqrt}(1.5)=1.22)$. By comparison, we think the amplifier slabs are $.35 / 1.6=5 \mathrm{X}$ below the PSD2 wavefront spec which translates to $25 \mathrm{X}$ below the scattered power spec. Consequently, we are inclined to believe that the curves in figure 4 agree to within our ability to characterize the PSD2 spectrum. A more mysterious feature of figure 4 , is the output energy scaling. As alluded to in figure 3, physical intuition would suggest that the back reflection sensor measurement should scale linearly with beam output energy. As shown in figure 4, the Prop92 model also predicts this, but the PBRS measurement does not. As of this date, no one seems to have provided a physical explanation for the concave shape of the measured PBRS curve. The Prop modeling and Stacie's time delay analysis suggest pinhole leakage as a likely explanation for the dominant PBRS blip, but further iterations on both the model and the data analysis are probably necessary.

In summary, we have provided a quantitative explanation for the dominant backreflection recorded in NEL experiments thus far. We feel that the first signal represents a measure of the PSD2 scattering efficiency in each laser chain. The second and third signals seem to correspond to pencil beams reflecting from SF1 and SF2. If this can be verified, these signals provide status information on the reflectivity of the AR coatings on these lenses. The final signal in figure 1 seems to be another cross-talk signal that must transit the switch in the 'off' state. As such, it can tell us how the PEPC extinction ratio is doing. In addition, signals from pinhole planes or from the vicinity of the target chamber will be of great importance to operations and have already been the subject of numerous FMEA calculations. Quantitative analysis of these reflections must be the first step toward automating their use in routine NIF operations. 